\title{
Strongly Hyperbolic Systems with Variable Coefficients
}

\author{
By \\ Kunihiko KajITANI*
}

\section{§1. Introduction}

We consider the first order partial differential equation in $R^{p} \times[0, T]$, $T>0$,

$$
L[u]=\frac{\partial}{\partial t} u-\sum_{j=1}^{p} A_{j}(x, t) \frac{\partial}{\partial x_{j}} u-B(x, t) u=f(x, t),
$$

where $A_{j}(x, t)$ and $B(x, t)$ are matrices of order $m$, infinitely differentiable with respect to $t$ and $x=\left(x_{1}, \ldots, x_{p}\right)$, and $u$ and $f$ are vector-valued functions with $m$ components. We consider the Cauchy problem for this equation with intial values given at $t=t_{0}>0$. We say that the Cauchy problem for (1.1) is uniformly well posed, if for any $f(x, t)$ infinitely differentiable and for any intial value $u\left(x, t_{0}\right)$ infinitely differentiable, there exists uniquely the infinitely differentiable solution $u(x, t)$ of (1.1) in $\Omega\left(x_{0}, t_{0}, \varepsilon\right)=\left\{(x, t) ;\left|x-x_{0}\right| \leqq \lambda_{0}\left(t_{0}+\varepsilon-t\right), t_{0} \leqq t \leqq t_{0}+\varepsilon\right\}$ for any $\varepsilon$, $0<\varepsilon \leqq \varepsilon_{0}$, where $\left(x_{0}, t_{0}\right)$ is an arbitrary point in $R^{p} \times[0, T]$ and $\lambda_{0}$ and $\varepsilon_{0}$ are positive constants. We denote by $L_{0}$ the principal part of $L$, that is, $L_{0}=\frac{\partial}{\partial t}-\sum A_{j}(x, t) \frac{\partial}{\partial x_{j}}$. We say that $L_{0}$ is strongly hyperbolic, if the Cauchy problem for (1.1) is uniformly well posed for any lower order $B(x, t)$ infinitely differentiable.

We suppose that the multiplicity of the characteristic roots is independent of $x, t$ and $\xi$. More precisely

$$
\operatorname{det}\left(\lambda-\sum A_{j}(x, t) \xi_{j}\right)=\prod_{j=1}^{l}\left(\lambda-\lambda_{j}(x, t: \xi)\right)^{{ }^{j}}
$$

Communicated by S. Matsuura, April 25, 1973.

* Department of Applied Mathematics and Physics, Kyoto University, Kyoto. 
where $\nu_{j}(j=1,2, \ldots, l)$ are positive integers independent of $(x, t, \xi)$ and

$$
\lambda_{i}(x, t: \xi) \neq \lambda_{j}(x, t: \xi) \quad(i \neq j) .
$$

Then we have

Theorem 1.1. Suppose that (1.2) and (1.3) are valid. Then $L_{0}$ is strongly hyperbolic, if and only if $\sum A_{j}(x, t) \xi_{j}$ is diagonalizable. ${ }^{1)}$

Remark 1. When the coefficients of $L$ are constant matrices, $\mathrm{K}$. Kasahara and M. Yamaguti proved in [7] that $L_{0}$ is strongly hyperbolic if and only if $\sum A_{j} \xi_{j}$ is uniformly diagonalizable.

Remark 2. In the case of variable coefficients, it is known that $\sum A_{j}(x, t) \xi_{j}$ is necessarily diagonalizable for $L^{2}$-well posedness of (1.1) (cf. [4], [6], [14]).

We note that the statements in Remark 1 and Remark 2 hold without the hypothesis that the multiplicity of characteristic roots is constant. But our Theorem 1.1 is not valid, if we do not assume that the multiplicity of characteristic roots is constant. For example,

$$
L_{0}=\frac{\partial}{\partial t}-\left(\begin{array}{cc}
t & 1 \\
0 & -t
\end{array}\right) \frac{\partial}{\partial x}
$$

is strongly hyperbolic, that is

$$
\left\{\begin{array}{l}
\left(L_{0}+B(x, t)\right) u(x, t)=f(x, t) \\
u(x, 0)=u_{0}(x)
\end{array}\right.
$$

is well posed for any lower order $2 \times 2$ matrix $B(x, t)$, inifinitely differentiable. We can see from Remark 2 that (1.4) is not $L^{2}$-well posed. But we have a positive integer $s_{0}$ such that there holds for any non-negative integer $k$,

$$
\|u(t)\|_{k} \leqq C\left\{\left\|u_{0}\right\|_{k+s_{0}}+\int_{0}^{t}\|f(s)\|_{k+s_{0}} d s\right\}, \text { 2) }
$$

1) We say that $\sum A_{j}(x, t) \xi_{j}$ is diagonalizable, if for any fixed point $(x, t, \xi)$ there exists a non-singular matrix $N$ so that $N\left(\sum \mathrm{A}_{j}(x, t) \xi_{j}\right) N^{-1}$ is a diagonal matrix.

2) This estimate is obtained by use of the result which O.A. Oleinik derived in [1]. 
where

$$
\|u(t)\|_{k}^{2}=\sum_{|\alpha|+j \leqq k} \int\left|\left(\frac{\partial}{\partial t}\right)^{j}\left(\frac{\partial}{\partial x}\right) u(x, t)\right|^{2} d x .
$$

In this example $L_{0}$ is not diagonalizable at $t=0$.

We say that the Cauchy problem for (1.1) with intial plane $t_{0}=0$ is $L^{2}$-well posed, if for any $u_{0}(x)$ in $L^{2}\left(R^{p}\right)$ given at $t=0$ and for any $f(x, t)$ in $\mathscr{E}_{t}^{0}\left(L^{2}\left(R^{p}\right)\right)$, there exists the solution $u(x, t)$ in $\mathscr{E}_{t}^{0}\left(L^{2}\left(R^{p}\right)\right)$ satisfying

$$
\|u(t)\| \leqq C\left\{\left\|u_{0}\right\|+\int_{0}^{t}\|f(s)\| d s\right\}, \quad \text { for } \quad t>0
$$

where $\|\cdot\|$ is the norm of $L^{2}\left(R^{p}\right)$.

Now we assume instead of (1.3),

$$
\inf _{\substack{(x, t) \in R^{p} \times[0, T] \\|\xi|=1, i \neq j}}\left|\lambda_{i}(x, t: \xi)-\lambda_{j}(x, t: \xi)\right| \geqq \delta>0 .
$$

Then it follows from Theorem 1.1 that,

Corollary. We suppose that (1.2) and (1.3)' are valid. Then the following statements are equivalent:

(i) $L_{0}$ is strongly hyperbolic.

(ii) $\sum A_{j}(x, t) \xi_{j}$ is diagonalizable.

(iii) The Cauchy problem (1.1) is $L^{2}$-well posed.

Theorem 1.1 implies (i) $\Rightarrow$ (ii). (iii) $\Rightarrow$ (i) is trivial. We note that T. Kano proved directly (iii) $\Rightarrow$ (ii) in [5].

Though S. Mizohata has proved already in [10] and [11] the sufficiency of Theorem 1.1 and (ii) $\Rightarrow$ (iii) in Corollary, we shall explain these facts. Namely, under the assumptions (1.2), (1.3) and the diagonalizability of $\sum A_{j}(x, t) \xi_{j}$, we can construct locally the symmetrizer of $\sum A_{j}(x, t) \xi_{j}$ and can derive the finite propagation speed of the solution of (1.1) and the existence of the solution of (1.1) for any $u_{0}$ and $f(x, t)$, in $L^{2}\left(R^{p}\right)$ and in $\mathscr{E}_{t}^{0}\left(L^{2}\left(R^{p}\right)\right)$, with compact supports, respectively. We put as the symmetrizer of $A(x, t: \xi)=\sum A_{j}(x, t) \xi_{j}$,

$$
\begin{gathered}
S(x, t: \xi)=\frac{1}{2 \pi i} \oint_{\Gamma}\left(\lambda-A(x, t: \xi)^{*}\right)^{-1}(\lambda-A(x, t: \xi))^{-1} \\
\times P_{\lambda}(x, t: \lambda, \xi)^{-1} P(x, t: \lambda, \xi) d \lambda,
\end{gathered}
$$


where $A(x, t: \xi)^{*}$ is the adjoint matrix of $A(x, t: \xi), P(x, t: \lambda, \xi)$ is the determinant of $(\lambda-A(x, t: \xi)), P_{\lambda}(x, t: \lambda, \xi)=\frac{\partial}{\partial \lambda} P(x, t: \lambda, \xi)$, and $\Gamma$ is a Jordan's curve which contains the characteristic roots of $A(x, t: \xi)$ and does not contain the poles of $P P_{\lambda}^{-1}(x, t: \lambda, \xi)$. Then $S(x, t: \xi)$ has the following properties: if $A(x, t: \xi)$ is diagonalizable and (1.2), (1.3) are valid,

$$
<S(x, t: \xi) h, h>\geqq \delta(x, t: \xi)|h|^{2},
$$

where, for any compact set $K$ in $R^{p}$,

$$
\inf _{\substack{(x, t) \in K \times[0, T] \\|\xi|=1}} \delta(x, t: \xi)=d(K)>0
$$

and

$$
(S(x, t: \xi) A(x, t: \xi))^{*}=S(x, t: \xi) A(x, t: \xi) .
$$

Moreover under the assumption $(1.3)^{\prime}$, we have

$$
\inf _{\substack{(x, t) \in R^{p}|\xi[0, T]\\| \xi \mid=1}} \delta(x, t: \xi)=d_{0}>0 .
$$

The proof of these properties is due to K.O. Friedrichs [3]. The suffciency of Theorem 1.1 can be proved by use of the properties (1.6), (1.7) and $(1.8)$ and $(\mathrm{ii}) \Rightarrow$ (iii) in Corollary by use of $(1.7)^{\prime}$ (See S. Mizohata [10], [11]).

In the next section we shall prove the necessity of Theorem 1.1 by use of the modified method which P.D. Lax introduced in [8].

Remark 3. For the single higher order equation $P\left(x, t: \frac{\partial}{\partial t}, \frac{\partial}{\partial x}\right)$ of homogeneous order $m$, it is known that the characteristic roots of $P(x, t ; \lambda, \xi)$ are real distinct, if and only if $P\left(x, t ; \frac{\partial}{\partial t}, \frac{\partial}{\partial x}\right)$ is strongly hyperbolic. This fact follows from the results which S. Mizohata and Y. Ohya in [12] and [13], and H. Flaschka and G. Strang in [2] proved.

\section{§2. Proof of Necessity of Theorem 1.1}

Here we shall prove that $\sum A_{j}(x, t) \xi_{i}$ is diagonalizable if $L_{0}$ is strongly hyperbolic. We need an inequality derived by the closed graph theorem. If the Cauchy problem (1.1) is uniformly well posed, it follows 
from the closed graph theorem that there exists a positive constant $C(\Omega)$ and a positive integer $s_{0}$ such that

$$
|u|_{0, \Omega} \leqq C(\Omega)\left\{|L u|_{s_{0}, \Omega}+|u|_{s_{0}, D}\right\},
$$

where $|\cdot|_{s, \Omega}$ is a supremum norm of $C^{s}(\Omega)$, and $\Omega=\left\{(x, t) ;\left|x-x_{0}\right| \leqq\right.$ $\left.\lambda_{0}\left(t^{\prime}-t\right), t_{0} \leqq t \leqq t^{\prime}\right\}$ and $D=\Omega \cap\left\{t=t_{0}\right\}$. We note that a constant $C(\Omega)$ may be generally dependent on $\Omega$. We put $\Omega_{\mu}=\left\{(x, t) ;\left|x-x_{0}\right| \leqq \lambda_{0}\left(t_{0}+\right.\right.$ $\left.\left.\frac{1}{\mu}-t\right), t_{0} \leqq t \leqq t_{0}+\mu^{-1}\right\}$ and $D_{\mu}=\Omega_{\mu} \cap\left\{t=t_{0}\right\}$. Then we have

Lemma 2.1. Suppose that the Cauchy problem of (1.1) is uniformly well posed. Then there exist positive integers $s_{0}, s_{1}$ and a constant $C_{0}$ such that it holds for any $\mu \geqq 1$ and for any $u(x, t)$ in $C^{\infty}\left(\Omega_{1}\right)$,

$$
|u|_{0, \Omega_{\mu}} \leqq C_{0} \mu^{s_{1}}\left\{|L u|_{s_{0}, \Omega_{\mu}}+|u|_{s_{0}, D_{\mu}}\right\},
$$

where $s_{0}, s_{1}$ and $C_{0}$ are independent of $\mu$.

Proof. Suppose that there exist functions $u_{\mu}(x, t)$ for any $\mu$ such that

$$
\left|u_{\mu}\right|_{0, \Omega_{\mu}} \geqq \mu^{s(\mu)}\left\{\left|L u_{\mu}\right|_{s_{0}, \Omega_{\mu}}+\left|u_{\mu}\right|_{s_{0}, D_{\mu}}\right\},
$$

where $s(\mu) \rightarrow \infty$ for $\mu \rightarrow \infty$. Without loss of generality we may assume $\left|u_{\mu}\right|_{0, \Omega_{\mu}}=1$. We put $L u_{\mu}=f_{\mu}$, and $u_{\mu}\left(x, t_{0}\right)=g_{\mu}$. We can extend $f_{\mu}(x, t)$ and $g_{\mu}(x)$ to the domain $\Omega_{1}=\left\{\left|x-x_{0}\right| \leqq \lambda_{0}\left(t_{0}+1-t\right), t_{0} \leqq t \leqq t_{0}+1\right\}$ such that

$$
\begin{aligned}
\tilde{f}_{\mu}(x, t) & =f_{\mu}(x, t) \quad \text { in } \quad \Omega_{\mu} \\
\tilde{g}_{\mu}(x)=g_{\mu}(x) & \text { in } \quad D_{\mu},
\end{aligned}
$$

and

$$
\left\{\begin{array}{l}
\left|\tilde{f}_{\mu}\right|_{s_{0}, \Omega_{1}} \leqq M^{2 \log _{2} \mu}\left|f_{\mu}\right|_{s_{0}, \Omega_{\mu}}, \\
\left|\tilde{g}_{\mu}\right|_{s_{0}, D_{1}} \leqq M^{\log _{2} \mu}\left|g_{\mu}\right|_{s_{0}, D_{\mu}},
\end{array}\right.
$$

where $M$ is independent of $\mu_{0}{ }^{3)}$ Let $\tilde{u}_{\mu}(x, t)$ be the solution of (1.1) for $\tilde{f}_{\mu}(x, t)$ and $\tilde{g}_{\mu}(x)$. Then the uniqueness of the solution implies

3) These inequalities will be proved in appendix. 


$$
\tilde{u}_{\mu}(x, t)=u_{\mu}(x, t) \quad \text { in } \quad \Omega_{\mu} .
$$

Hence we have

$$
\left|\tilde{u}_{\mu}\right|_{0, \Omega_{1}} \geqq 1 \text {. }
$$

On the other hand, by virtue of (2.1), we have

$$
\left|\tilde{u}_{\mu}\right|_{0, \Omega_{1}} \leqq C\left(\Omega_{1}\right)\left(\left|\tilde{f}_{\mu}\right|_{s_{0}, \Omega_{1}}+\left|\tilde{g}_{\mu}\right|_{s_{0}, D_{1}}\right),
$$

from which we get, combining with (2.3) and (2.4), the following inequality

$$
\left|\tilde{u}_{\mu}\right|_{0, \Omega_{1}} \leqq C\left(\Omega_{1}\right)\left(M^{2 \log _{2} \mu}\right) \mu^{-s(\mu)} .
$$

This and (2.5) cannot be compatible if $\mu$ is sufficiently large.

Now, we shall prove our theorem by contradiction. Assume that $\sum A_{j}(x, t) \xi_{j}$ is not diagonalizable. Then for some $B(x, t)$, the Cauchy problem for differential operator $L_{0}+B(x, t)$ can not be uniformly well posed.

Lemma 2.2. Assume that (1.2) and (1.3) are valid. Then there exist an open set $U_{0}$ in $R^{p} \times[0, T] \times\left(R^{p}-\{0\}\right)$ and a non-singular matrix $N(x, t ; \xi)$, of which elements are as smooth as those of $A(x, t ; \xi)$ in $U_{0}$, such that,

$$
N(x, t: \xi) A(x, t: \xi)=\left(\begin{array}{llll}
D_{1} & & & \\
& D_{2} & & \\
& & \ddots & \\
0 & & & D_{k}
\end{array}\right) N(x, t: \xi)
$$

where $D_{i}$ are $m_{i} \times m_{i}$ Jordan's forms, that is,

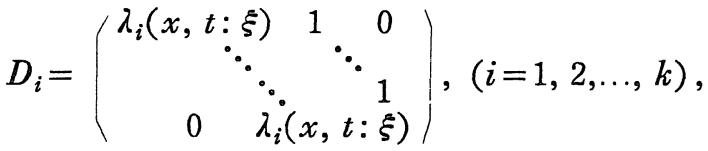

$$
\begin{aligned}
& \sum m_{i}=m .
\end{aligned}
$$

We shall prove this lemma in the appendix.

Remark. It is known that characteristic roots $\lambda_{i}(x, t: \xi)$ of $A(x, t: \xi)$ 
are real, if the Cauchy problem of (1.1) is uniformly well posed (cf. S. Mizohata [9]).

Now we assume that $A(x, t: \xi)$ is not diagonalizable, that is, for some $i_{0}$ we have $m_{i_{0}} \geqq 2$. We may assume $i_{0}=1$ without loss of generality. Moreover we may assume that an open set $U_{0}$ in Lemma 2.2 is a neighbourhood of $\left(0,0, \xi_{0}\right)$. We note that characteristic roots $\lambda_{i}(x, t: \xi)$ are analytic with respect to $\xi$. We write $\lambda_{1}(x, t: \xi)=a(x, t: \xi)$. Then we can find an analytic function $\lambda^{0}(x, t)$ (real valued) satisfying

$$
\begin{aligned}
& \lambda_{t}^{0}(x, t)=\sum_{j+|\nu| \leqq r_{0}}(j ! \nu !)^{-1}\left(t^{j} x^{\nu}\right)\left(\frac{\partial}{\partial t}\right)^{j}\left(\frac{\partial}{\partial x}\right)^{\nu} a\left(0,0 ; \lambda_{x}^{0}\right) \\
& \lambda^{0}(x, 0)=x \cdot \xi_{0}
\end{aligned}
$$

Then we have

$$
\left\{\begin{array}{l}
\lambda_{t}^{0}=a\left(x, t: \lambda_{x}^{0}\right)+O\left(x^{\nu_{0}} t^{j_{0}}\right), \nu_{0}+j_{0}=r_{0}+1 \\
\lambda^{0}(x, 0)=x \cdot \xi_{0}
\end{array}\right.
$$

We put $N_{0}(x, t)=N\left(x, t: \lambda_{x}^{0}(x, t)\right)$. Then we note that

$$
N_{0}(x, t) A\left(x, t: \lambda_{x}^{0}\right) N_{0}^{-1}(x, t)=\left(\begin{array}{cc}
D_{1} & 0 \\
0 & \check{D}
\end{array}\right)+O\left(x^{\nu_{0}} t^{j_{0}}\right)
$$

where

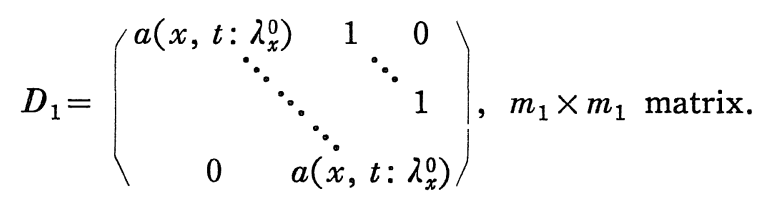

We transform $L$ by $N_{0}(x, t)$. We put $v=N_{0} u$. Then we have

$$
\begin{aligned}
\tilde{L}[v]= & \frac{\partial}{\partial t} v-\sum_{j} \tilde{A}_{j}(x, t) \frac{\partial}{\partial x_{j}} v+\sum_{j} N_{0} A_{j} N_{0 x_{j}}^{-1} v \\
& +N_{0} N_{0 t}^{-1} v-N_{0} B N_{0}^{-1} v,
\end{aligned}
$$

where

$$
\tilde{A}_{j}(x, t)=N_{0} A_{j}(x, t) N_{0}^{-1} .
$$


Now we choose $B(x, t)$ such that

$$
N_{0} A_{j} N_{0 x_{j}}^{-1}-N_{0} N_{0 t}^{-1}-N_{0} B N_{0}^{-1}=B_{0}
$$

where $\left(m_{1}, 1\right)$ element of $B_{0}$ is -1 and the other elements are zero. We consider the Cauchy problem for $\tilde{L}$, that is,

$$
\left\{\begin{array}{l}
\tilde{L}[v]=\left(\frac{\partial}{\partial t}-\sum \tilde{A}_{j}(x, t) \frac{\partial}{\partial x_{j}}+B_{0}\right) v(x, t)=f(x, t) \\
v(x, 0)=v_{0}(x)
\end{array}\right.
$$

Then the Cauchy problem for (2.10) is uniformly well posed, if the Cauchy problem for $L$ is uniformly well lposed. Hence the inequality (2.2) with $\left(x_{0}, t_{0}\right)=(0,0)$ holds for $\tilde{L}$, that is, for any $\mu \geqq 1$,

$$
|v|_{0, \Omega_{\mu}} \leqq C_{0} \mu^{s_{1}}\left\{|\tilde{L} v|_{s_{0}, \Omega_{\mu}}+|v|_{s_{0}, D_{\mu}}\right\}
$$

Our purpose is to construct the asymptotic solution $\{v(x, t)\}$ of $(2.10)$ with $\tilde{f}=0$ and $v_{0}=0$, which violates the inequality (2.11).

We construct the asymptotic solution of the following form,

$$
v(x, t: n)=\sum_{j \geqq 0} e^{i n \lambda(x, t: n)} h_{j} n^{-j / m_{1}},
$$

where $h_{j}$ are constant vectors of forms ${ }^{t}\left(h_{j}^{(1)}, \ldots, h_{j}^{\left(m_{1}\right)}, 0, \ldots, 0\right), h_{j}^{(i)}(i=1 \ldots$ $\left.m_{1}\right)$ being constant numbers and $\lambda(x, t: n)$ is of form as

$$
\lambda(x, t: n)=\sum_{j \geqq 0}\left(\lambda^{0}(x, t)+\sigma_{j} t\right) n^{-j / m_{1}},\left(\sigma_{j} \text { constant. }\right)
$$

We write,

$$
\begin{aligned}
& h(n)=\sum_{j \geq 0} h_{j} n^{-j / m_{1}}, \\
& \sigma(n)=\sum_{j \geq 0} \sigma_{j} n^{-j / m_{1}}, \\
& e(n)=\sum_{j \geq 0} n^{-j / m_{1}},
\end{aligned}
$$

and

$$
h^{0}(n)=\sum_{j \geqq 0}{ }^{t}\left(h_{j}^{(1)}, \ldots, h_{j}^{\left(m_{1}\right)}\right) n^{-j / m_{1}}
$$

Applying $\tilde{L}$ to $v$ defined in (2.12) we have 


$$
\tilde{L}[v]=i n\left(\lambda_{t}(x, t: n)-\sum_{k=1}^{p} \tilde{A}_{k} \lambda_{x_{k}}(x, t: n)-\frac{i}{n} B_{0}\right) h(n)
$$

$$
=i n\left(\begin{array}{c}
\Lambda(n) h^{0}(n) \\
0
\end{array}\right)+O\left(n x^{\nu_{1}} t^{j_{1}}\right), \quad\left|\nu_{1}\right|+j_{1}=r_{0}
$$

where $i=\sqrt{-1}$, and

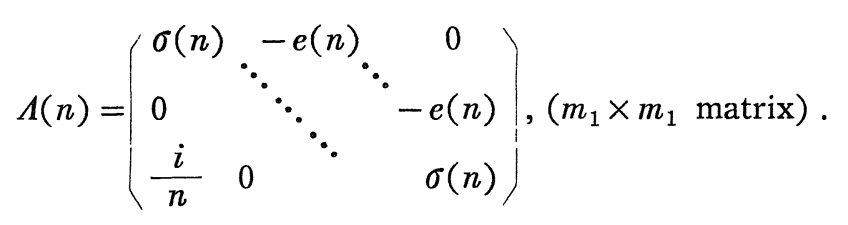

We determine $\sigma(n)$ such that $\operatorname{det} \Lambda(n)=0$, that is,

$$
\begin{aligned}
\operatorname{det} \Lambda(n) & =\sigma(n)^{m_{1}}+\frac{i}{n} e(n)^{m_{1}-1} \\
& =\left(\sum \sigma_{j} n^{-j / m_{1}}\right)^{m_{1}}+\frac{i}{n}\left(\sum n^{-j / m_{1}}\right)^{m_{1}-1} \\
& =\sum_{j \geqq 0} F_{j} n^{-j / m_{1}}
\end{aligned}
$$

where

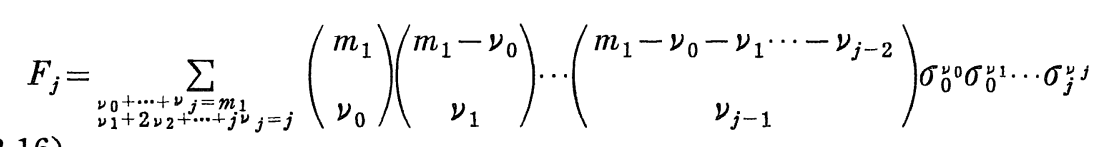

$$
+i \sum_{\substack{\nu_{0}+\cdots+\nu_{j}=m_{1}-1 \\
\nu_{1}+2 \nu_{2}+\cdots+\left(j-m_{1}\right)_{\nu j-m_{1}}=j-m_{1}}}\left(\begin{array}{c}
m_{1}-1 \\
\nu_{0}
\end{array}\right) \cdots\left(\begin{array}{c}
m_{1}-1-\nu_{0}-\cdots-\nu_{j-2} \\
\nu_{j-1}
\end{array}\right) .
$$

From this formula, we have $F_{0}=\sigma_{0}^{m_{1}}$. Hence we have

$$
\sigma_{0}=0
$$

which implies $F_{j}=0\left(j<m_{1}\right)$. Next, we obtain

$$
F_{m_{1}}=\sigma_{1}^{m_{1}}+i=0
$$

Hence we can choose $\sigma_{1}$ such that

$$
\operatorname{Im} \sigma_{1}<0 \text {. }
$$


As $\sigma_{0}$ is zero, we have generally from (2.16)

$$
F_{j}=m_{1} \sigma_{1}^{m_{1}-1} \sigma_{j-m_{1}+1}+P_{j}\left(\sigma_{1}, \ldots, \sigma_{j-m_{1}}\right)=0, j>m_{1},
$$

where $P_{j}$ is polynomials of $\left(\sigma_{1}, \ldots, \sigma_{j-m_{1}}\right)$. Hence we can determine $\sigma_{j}$ so that (2.18) holds.

Next we determine $h^{0}(n)$ such that $\Lambda(n) h^{0}(n)=0$. As we can write

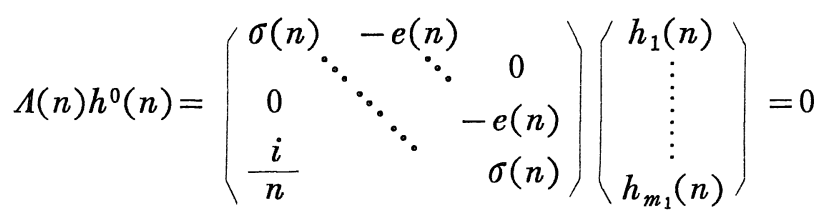

we obtain the relations,

$$
h_{k}(n)=\left(\frac{e(n)}{\sigma(n)}\right)^{m_{1}-k} h_{m_{1}}(n), k=1, \ldots, m_{1}-1
$$

We choose $h_{m_{1}}(n)=\sigma(n)^{m_{1}-1}$. Then we have $h_{k}(n)=e(n)^{m_{1}-k} \sigma(n)^{k-1}$. Hence we can expand $h^{0}(n)$ as

$$
h^{0}(n)=\sum_{j \geqq 0} h_{j}^{0} n^{-j / m_{1}},
$$

where $h_{j}^{0}$ are constant vectors. Here we note

$$
h_{0}^{0}={ }^{t}(1,0, \ldots, 0) \neq 0 \text {. }
$$

Now, we define $v_{N}(x, t: n)$ as

$$
v_{N}(x, t: n)=\sum_{j=0}^{N} e^{i n \lambda_{N}(x, t: n)} h_{j} n^{-j / m_{1}},
$$

where

$$
\lambda_{N}(x, t: n)=\sum_{j=0}^{N}\left(\lambda^{0}(x, t)+\sigma_{j} t\right) n^{-j / m_{1}}
$$

and $h_{j}={ }^{t}\left(h_{j}^{0}, 0, \ldots, 0\right)$. Then we have

$$
L\left[v_{N}\right]=e^{i n \lambda_{N}}\left(O\left(n^{-N_{1}}\right)+O\left(n x^{\nu_{1}} t^{j_{1}}\right)\right),\left|\nu_{1}\right|+j_{1}=r_{0}
$$

where $N_{1}$ becomes larger and larger if we choose $N$ larger and larger. We put 


$$
\rho(n, \mu)=\max _{(x, t) \in \bar{\Omega}_{\mu}}\left\{-\operatorname{Im} n \lambda_{N}(x, t: n)\right\} .
$$

Then by virtue of (2.17), we obtain

$$
\rho(n, \mu) \geqq c n^{1-1 / m_{1}} \mu^{-1}
$$

where $c$ is independent of $n$ and $\mu$. Here we choose $\mu=n^{\left(m_{1}-1\right) / 2 m_{1}}$. Then we have

$$
\rho(n, \mu)>c n^{\left(m_{1}-1\right) / 2 m_{1}} .
$$

From (2.21), we obtain

$$
\left|L\left[v_{N}\right]\right|_{s_{0}, \Omega_{\mu}} \leqq \text { const. } e^{\mu(n, \mu)}\left(n^{-N_{1}+s_{0}}+n^{s_{0}+1} \mu^{-r_{0}+s_{0}}\right)
$$

where const. is independent of $n$ and $\mu$ and $r_{0}=\nu_{1}+j_{1}$. Noting that $\operatorname{Im} \lambda_{N}(x, 0 ; n)=0$, we have

$$
\left|v_{N}\right|_{s_{0}, D_{\mu}} \leqq \text { const. } n^{s_{0}} \text {. }
$$

On the other hand we obtain by virtue of $(2.20)$,

$$
\left|v_{N}\right|_{0, \Omega_{\mu}} \geqq c_{1} e^{\rho(n, \mu)} \text {. }
$$

Hence it follows from (2.11), (2.23), (2.24) and (2.25) that

$$
\begin{gathered}
c_{1} e^{\rho(n, \mu)} \leqq \text { const. }\left\{e^{\rho(n, \mu)}\left(n^{s_{0}-N_{1}}+n^{s_{0}+1-\left(r_{0}-s_{0}\right)\left(m_{1}-1\right) / 2 m_{1}}\right)\right. \\
\left.+n^{s_{0}}\right\} n^{s_{1}\left(m_{1}-1\right) / 2 m_{1}} .
\end{gathered}
$$

Here we choose $N_{1}$ and $r_{0}$ such that $N_{1}=s_{0}+1+s_{1}\left(m_{1}-1\right) / 2 m_{1}$ and $s_{0}+1-\left(r_{0}-s_{0}\right)\left(m_{1}-1\right) / 2 m_{1}=-1-s_{1}\left(m_{1}-1\right) / 2 m_{1}$. Then (2.26) and (2.22) can not be compatible, if $n$ is sufficiently large. This proves the necessity of our Theorem 1.1.

\section{Appendix}

Lemma A.1. Let $g(x)$ be in $C^{s_{0}}\left(D_{\mu}\right)$, where $D_{\mu}=\left\{x \in R^{p},|x| \leqq \mu^{-1}\right\}$, $\mu \geqq 1$. Then there exists $\tilde{g}(x)$ in $C^{s_{0}}\left(D_{1}\right)$ such that

$$
\tilde{g}(x)=g(x) \quad \text { in } D_{\mu} \text {, }
$$

and 


$$
|\tilde{g}|_{s_{0}, D_{1}} \leqq M^{\log _{2} \mu}|g|_{s_{0}, D_{\mu}}
$$

where $M$ is independent of $\mu$.

Proof. Under the change of variables, $|x|=r$, the domain $D_{\mu}$ is transformed into the domain $\widetilde{D}_{\mu}=\left\{(r, \omega), 0 \leqq r \leqq \mu^{-1}, \omega \in S^{p-1}\right\}$, where $S^{p-1}$ is the unit sphere. And $g(x)$ is transformed into $g(r, \omega)$ in $C^{s_{0}}\left(\tilde{D}_{\mu}\right)$. We extend $g(r, \omega)$ to the domain $D_{\mu}^{(1)}=\left\{(r, \omega), 0 \leqq r \leqq 2 \mu^{-1}, \omega \in S^{p-1}\right\}$,

$$
\begin{aligned}
g^{(1)}(r, \omega) & =g(r, \omega), 0 \leqq r \leqq \mu^{-1}, \omega \in S^{p-1} \\
& =\sum_{i=1}^{s 0+1} a_{i} g\left(\mu^{-1}-\frac{i}{s_{0}+1}\left(r-\mu^{-1}\right), \omega\right), \mu^{-1}<r \leqq 2 \mu^{-1},
\end{aligned}
$$

where $\sum_{i=1}^{s 0+1} a_{i}\left(\frac{i}{s_{0}+1}\right)^{j}=1, j=0,1, \ldots, s_{0}$. Then $g^{(1)}(r, \omega)$ belongs to $C^{s_{0}}\left(D_{\mu}^{(1)}\right)$ and satisfies

$$
\left|g^{(1)}\right|_{s_{0}, D_{\mu}^{(1)}} \leqq M|g|_{s_{0}, D_{\mu}} \text {. }
$$

Next, we extend $g^{(1)}(r, \omega)$ to a domain $D_{\mu}^{(2)}=\left\{(r, \omega), 0 \leqq r \leqq 4 \mu^{-1}, \omega \in S^{p-1}\right\}$,

$$
\begin{aligned}
g^{(2)}(r, \omega)= & g^{(1)}(r, \omega), 0 \leqq r \leqq 2 \mu^{-1}, \\
= & \sum_{i=1}^{s 0+1} a_{i} g^{(1)}\left(2 \mu^{-1}-\frac{i}{s_{0}+1}\left(r-2 \mu^{-1}\right), \omega\right), \\
& 2 \mu^{-1}<r<4 \mu^{-1} .
\end{aligned}
$$

Generally, for any positive integer $m$ we define $g^{(m+1)}(r, \omega)$ as

$$
\begin{aligned}
& g^{(m+1)}(r, \omega)=g^{(m)}(r, \omega), 0 \leqq r \leqq 2^{m} \mu^{-1} \\
&= \sum_{i=1}^{s+1} a_{i} g^{(m)}\left(2^{m} \mu^{-1}-\frac{i}{s_{0}+1}\left(r-2^{m} \mu^{-1}\right), \omega\right) \\
& 2^{m} \mu^{-1}<r \leqq 2^{m+1} \mu^{-1} .
\end{aligned}
$$

Then $g^{(m+1)}(r, \omega)$ belongs to $C^{s_{0}}\left(D_{\mu}^{(m+1)}\right)$, where $D^{(m+1)}=\{(r, \omega), 0 \leqq r \leqq$ $\left.2^{m+1} \mu^{-1}, \omega \in S^{p-1}\right\}$, and satisfies

$$
\begin{aligned}
\left|g^{(m+1)}\right|_{s_{0}, D_{\mu}^{(m+1)}} \leqq M\left|g^{(m)}\right|_{s_{0}, D_{\mu}^{(m)}} \\
\leqq M^{m+1}|g|_{s_{0}, D_{\mu}} .
\end{aligned}
$$


Hence, if we put $m+1=\log _{2} \mu$, we have $D^{(m+1)}=D_{1}$ and $\tilde{g}(x)=g^{(m+1)}(r, \omega)$ satifying (A.1) and (A.2).

Q.E.D.

Corollary. Let $f(x, t)$ be in $C^{s}\left(\Omega_{\mu}\right)$, where $\Omega_{\mu}=\left\{(x, t),|x|<\lambda_{0}\right.$ $\left.\left(\mu^{-1}-t\right), 0 \leqq t \leqq \mu^{-1}\right\}, \mu \geqq 1$. Then there exists $\tilde{f}(x, t)$ in $C^{s_{0}}\left(\Omega_{1}\right)$ such that

and

$$
\tilde{f}(x, t)=f(x, t) \text { in } \Omega_{\mu},
$$

$$
|\tilde{f}|_{s_{0}, \Omega_{1}}<M^{2 \log _{2} \mu}|f|_{s_{0}, \Omega_{\mu}}
$$

We can prove this corollary by the same method as in Lemma A.1, if we change variables $(x, t)$ into $(r, \omega, \tau)$ as $r=|x|, \tau=\mu^{-1}-t-r$.

Lemma A.2. Let $A(z)$ be a $m \times m$ matrix of which elements are infinitely differentiable functions of $z$ in an open set $B_{1}$ in $R^{n}$ and of which eigenvalues are all zero. Then there exists a non-singular matrix $N(z)$ of which elements are infinitely differentiable in an open set $B \subset B_{1}$, such that

$$
N(z)^{-1} A(z) N(z)=\left(\begin{array}{llll}
J_{1} & & & 0 \\
& J_{2} & & \\
& & \ddots & \\
0 & & & J_{l}
\end{array}\right), \quad \text { for } z \text { in } B
$$

where

$$
J_{i}=\left(\begin{array}{cccc}
0 & 1 & & 0 \\
& \ddots & \ddots & \\
& & \ddots & 1 \\
0 & & & 0
\end{array}\right)
$$

Proof. Since the eigenvalues of $A(z)$ are all zero, there exists an integer $\nu(z)$ for any $z$ such that $A(z)^{\nu}=0$ and $A(z)^{\nu-1} \neq 0$. We put

$$
\nu_{0}=\max _{z \in \bar{B}_{1}} \nu(z)=\nu\left(z_{0}\right)
$$

Then we have a neighbourhood $B_{2}$ of $z_{0}$ in $B_{1}$ such that $A(z)^{\nu_{0}}=0$ and $A(z)^{\nu_{0}-1} \neq 0$ for any $z$ in $B_{2}$. Moreover we have an open set $B_{3}$ in $B_{2}$ such that the rank of $A(z)^{i}$ is constant for any $i=1,2, \ldots, \nu_{0}-1$ and for 
any $z$ in $B_{3}$. We define $W_{i}(z)$ as

$$
W_{i}(z)=\left\{h \in C^{m}, A(z)^{i} h=0\right\} .
$$

Then we note that $\operatorname{dim} W_{i}(z)=m_{i}$ is constant for $z$ in $B_{3}$ and

$$
C^{m}=W_{\nu_{0}}(z) \supset W_{\nu_{0}-1}(z) \supset \cdots \supset W_{1}(z)
$$

We put $r_{i}=m_{i}-m_{i-1}\left(i=2, \ldots, \nu_{0}\right)$ and $r_{1}=m_{1}$. Then there exist constant vectors $\left\{h_{1}, \ldots, h_{r_{\nu 0}}\right\}$ such that

$$
W_{\nu 0}(z)=\left\{h_{1}, \ldots, h_{r_{\nu 0}}\right\}+W_{\nu_{0}-1}(z) \quad \text { (direct sum) }
$$

for any $z$ in $B_{4} \subset B_{3}$, where $B_{4}$ is some open set in $B_{3}$. Then we have $A(z) h_{j}$ in $W_{\nu_{0}-1}(z)$ and $\left\{A(z) h_{1}, \ldots, A(z) h_{r_{\nu 0}}\right\} \cap W_{\nu_{0}-2}(z)=\{0\}$ for any $z \in B_{4}$. Hence we have $r_{\nu_{0}} \leqq r_{\nu_{0}-1}$. Hence there exsit constant vectors $\left\{h_{r_{\nu 0}+1}, \ldots, h_{r_{\nu 0-1}}\right\}$ such that

$$
\begin{aligned}
W_{\nu_{0}-1}(z)= & \left\{h_{1}, \ldots, A h_{r_{\nu 0}}, h_{r_{\nu 0}+1}, \ldots, h_{r_{\nu 0-1}}\right\} \\
& +W_{\nu_{0}-2}(z) \quad \text { (direct sum) }
\end{aligned}
$$

for any $z$ in $B_{5} \subset B_{4}$, where $B_{5}$ is some open set in $B_{4}$. Then we have $A(z)^{2} h_{j}\left(j=1, \ldots, r_{\nu_{0}}\right)$ and $A(z) h_{j}\left(j=r_{\nu_{0}}+1, \ldots, r_{\nu_{0}-1}\right)$ in $W_{\nu_{0}-2}(z)$ and $\left\{A(z)^{2} h_{1}, \ldots, A(z)^{2} h_{r_{\nu 0}}, A(z) h_{r_{\nu 0}+1}, \ldots, A(z) h_{r_{\nu 0-1}}\right\} \cap W_{\nu_{0}-3}(z)=\{0\}$ for $z$ in $B_{5}$. Hence we have $r_{\nu_{0}-1} \leqq r_{\nu_{0}-2}$. Similarly, we have $r_{\nu_{0}} \leqq r_{\nu_{0}-1} \leqq \cdots$ $\leqq r_{1}$. Therefore we can choose constant vectors $h_{1}, \ldots, h_{r_{1}}$ such that, $i=1, \ldots, \nu_{0}$

$$
\begin{aligned}
W_{i}(z)= & \left\{A(z)^{\nu_{0}-i} h_{1}, \ldots, A(z)^{\nu_{0}-i} h_{r_{\nu 0}}, A(z)^{\nu_{0}-i-1} h_{r_{\nu 0}+1}, \ldots,\right. \\
& \left.A(z)^{\nu_{0}-i-1} h_{r_{\nu 0-1}}, \ldots, h_{r_{i+1}+1}, \ldots, h_{r_{i}}\right\} \\
& +W_{i-1}(z) \quad(\text { direct } \text { sum })
\end{aligned}
$$

for $z$ in $B_{4+i} \subset B_{4+i-1}$, where $B_{4+i}$ is some open set in $B_{4+i-1}$. Then $A(z)^{k} h_{r_{i+1}+1}, \ldots, A(z)^{k} h_{r_{i}},\left(i=1,2, \ldots, \nu_{0} ; k=0,1, \ldots, i-1\right)$ become the basis of $W_{\nu_{0}}(z)=C^{m}$ for $z$ in $B_{3+\nu_{0}}$

$$
N_{j}^{(i)}(z)=\left(h_{j}, A(z) h_{j}, \ldots, A(z)^{i-1} h_{j}\right), j=r_{i+1}, \ldots, r_{i} .
$$

Then we obtain 


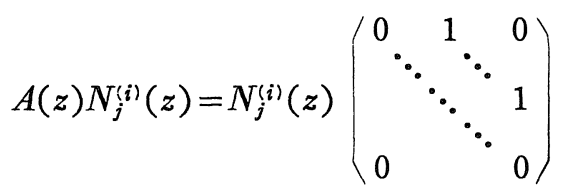

We put

$$
N(z)=\left(N_{1}^{\left(\nu_{0}\right)}, \ldots N_{r_{\nu 0}}^{\left(\nu_{\nu}\right)}, N_{r_{\nu 0}+1}^{\left(\nu_{0}-1\right)}, \ldots, N_{r_{\nu_{0}-1}}^{\left(\nu_{0}-1\right)}, \ldots, N_{r_{2}+1}^{(0)}, \ldots, N_{r_{1}}^{(0)}\right)
$$

Then $N(z)$ satisfies (A.3) for $z$ in $B=B_{\nu_{0}+3}$.

Proof of Lemma 2.2. For the fixed point $z_{0}=\left(x_{0}, t_{0}, \xi_{0}\right)$ we denote by $\left\{h_{1}^{(i)}, \ldots, h_{m_{i}}^{(i)}\right\}$ the basis of the generalized eigenspace of $A\left(z_{0}\right)$ corresponding to the eigen value $\lambda_{i}\left(z_{0}\right)$ of $A\left(z_{0}\right)$. We put

$$
h_{j}^{(i)}(z)=\frac{1}{2 \pi \sqrt{-1}} \oint_{\left|\lambda-\lambda_{i}\left(z_{0}\right)\right|=r_{0}}(\lambda-A(z))^{-1} h_{j}^{(i)} d \lambda, \quad\left(j=1, \ldots, m_{i}\right) .
$$

Then $\left\{h_{1}^{(i)}(z), \ldots, h_{m_{1}}^{(i)}(z)\right\}$ constructs the basis of the generalized root space of $A(z)$ corresponding to $\lambda_{i}(z)$ the eigenvalue of $A(z)$, for $z$ in a neighbourhood of $z_{0}$. We put $N_{1}(z)=\left(h_{1}^{(1)}(z), \ldots, h_{m_{1}}^{(1)}(z) \ldots, h_{1}^{(l)}(z), \ldots, h_{m}^{(l)}(z)\right)$. Then we have

$$
N_{1}(z) A(z) N_{1}(z)^{-1}=\left(\begin{array}{rrr}
A_{1}(z) & & 0 \\
& A_{2}(z) & \\
& \ddots & \\
0 & & A_{l}(z)
\end{array}\right) \text {, }
$$

where $A_{i}(z)$ has only the eigenvalue $\lambda_{i}(z)$. Hence we can apply Lemma A.2 to $\left(\lambda_{i}(z)-A_{i}(z)\right)$ for $z$ in $B_{0}$.

\section{References}

[1] Oleinik, O.A., On the Cauchy problem for weakly hyperbolic equations, Comm. Pure Apl. Math. 28 (1970) 569-586.

[2] Flaschka, H. and Strang, G., The correctness of the Cauchy problem, Advanced in Math. 6 (1971), 347-379.

[3] Friedrichs, K.O., Pseudo-differential operators, Lecture Note in Courant Institute (1968-'69).

[4] Kajitani, K., A necessary condition for the $L^{2}$-well posed Cauchy problem with variable coefficients, J. Math. Kyoto Univ. 13 (1973), 309-310. 
[5] Kano, T., A necessary condition for the well-posedness of the Cauchy problem for the first order hyperbolic system with multiple characteristics, Publ. RIMS, Kyoto Univ. 5 (1969), 149-164.

[6] Kano, T., Les système strictement hyperboliques, Comptes rendus, série A 274 (1972), 1116-1119.

[7] Kasahara, K. and Yamaguti, M., Strongly hyperbolic systems of linear partial differential equations with constant coefficients, Mem. Coll, Sci. Kyoto Univ. Ser. A 33 (1960), 1-23.

[8] Lax, P.D., Asymptotic solutions of oscillatory initial value problems, Duke Math. J. 24 (1957), 627-646.

[9] Mizohata, S., Some remarks on the Cauchy problem, J. Math. Kyoto Univ. 1 (1961), 109-127.

[10] Mizohata, S., Systèmes hyperboliques, J. Math. Soc. Japan 11 (1959), 205-233.

[11] Mizohata, S., Le problème de Cauchy pour les systèmes hyperboliques et paraboliques, Mem. Coll. Sci. Kyoto Univ., Ser. A, 22 Math. (1959), 181-212.

[12] Mizohata, S., and Ohya, Y., Sur la condition de E.E. Levi concernant des équations hyperboliques, Publ. RIMS Kyoto Univ. 4 (1968) 511-526.

[13] Mizohata, S., and Ohya, Y., Sur la condition d'hyperbolicité pour les équations à caracteristiques multiples II, Japanese J. Math. 40 (1971), 63-104.

[14] Strang, G., Necessary and insufficient conditions for well-posed Cauchy Problem, J. Diff. Eq. 2 (1966), 107-114. 